\title{
THE VANISHING VISCOSITY METHOD IN ONE-DIMENSIONAL THERMOELASTICITY
}

\author{
GUI-QIANG CHEN AND CONSTANTINE M. DAFERMOS
}

\begin{abstract}
The vanishing viscosity method is applied to the system of conservation laws of mass, momentum, and energy for a special class of one-dimensional thermoelastic media that do not conduct heat. Two types of vanishing "viscosity" are considered: Newtonian and artificial, in both cases accompanied by vanishing heat conductivity. It is shown that in either case one can pass to the zero viscosity limit by the method of compensated compactness, provided that velocity and pressure are uniformly bounded. Oscillations in the entropy field may propagate along the linearly degenerate characteristic field but do not affect the compactness of the velocity field or the pressure field. A priori bounds on velocity and pressure are established, albeit only for the case of artificial viscosity.
\end{abstract}

\section{INTRODUCTION}

The balance laws of mass, momentum, and energy for one-dimensional elastic media that do not conduct heat are expressed, in Lagrangian coordinates, by the equations

$$
\left\{\begin{array}{l}
\partial_{t} v-\partial_{x} u=0, \\
\partial_{t} u+\partial_{x} p=0, \\
\partial_{t}\left(e+\frac{1}{2} u^{2}\right)+\partial_{x}(u p)=0,
\end{array}\right.
$$

where $v, u, p$, and $e$ denote respectively deformation gradient (specific volume for fluids, strain for solids), velocity, pressure, and internal energy. Other relevant fields are the entropy $s$ and the temperature $\theta$.

The above system of conservation laws is complemented by the Clausius inequality

$$
\partial_{t} s \geq 0
$$

which expresses the second law of thermodynamics.

Received by the editors January 24, 1994.

1991 Mathematics Subject Classification. Primary 35L65, 35D05; Secondary 35A35.

Key words and phrases. Thermoelasticity, viscosity method, compensated compactness.

The first author was supported in part by the National Science Foundation, under grant \# DMS9207080, in part by the Office of Naval Research under contract \# N00014-91-J-1384, and in part by the Alfred P. Sloan Foundation, and the second author was supported in part by the National Science Foundation, under grant \# DMS-9208284, in part by the Army Research Office under contract \# DAAH04-93-G-0125, and in part by the Office of Naval Research under contract \# N00014-92-J-1481. 
Selecting $(v, u, s)$ as the state vector, we write constitutive equations

$$
e=\hat{e}(v, s), \quad p=\hat{p}(v, s), \quad \theta=\hat{\theta}(v, s)
$$

satisfying the conditions

$$
\hat{p}=-\hat{e}_{v}, \quad \hat{\theta}=\hat{e}_{s},
$$

whose role is to ensure that (1.2) holds automatically (as an equality) for any smooth solution of (1.1).

Under the standard assumptions $\hat{p}_{v}<0$ and $\hat{\theta}>0$, the system (1.1) is strictly hyperbolic. As is well known, solutions of the initial value problem, starting out from smooth initial data, eventually develop discontinuities that propagate as shock waves. Thus only a theory of weak solutions in the large maybe feasible. In the context of weak solutions, (1.2) is no longer a consequence of (1.1),(1.3), and (1.4) but rather an independent condition identifying physically admissible solutions of (1.1).

When the initial data have small total variation, a global BV solution of (1.1) can be constructed by the random choice method [Gl, Li]. However, the issue of existence of solutions with "large" initial data is still unresolved. One may attempt to construct solutions with possibly large oscillation by the method of "vanishing viscosity" in which the elastic nonconductor of heat is visualized as the $\mu \downarrow 0$ limit of a family of viscoelastic media with viscosity $\mu \kappa$ and heat conductivity $\mu \nu$, where $\mu>0$ and $\kappa=\hat{\kappa}(v, s) \geq 0, \nu=\hat{\nu}(v, s) \geq 0$. The balance laws of mass, momentum, and energy for these media read

$$
\left\{\begin{array}{l}
\partial_{t} v-\partial_{x} u=0, \\
\partial_{t} u+\partial_{x} p=\mu \partial_{x}\left(\kappa \partial_{x} u\right), \\
\partial_{t}\left(e+\frac{1}{2} u^{2}\right)+\partial_{x}(u p)=\mu \partial_{x}\left(\kappa u \partial_{x} u\right)+\mu \partial_{x}\left(\nu \partial_{x} \theta\right) .
\end{array}\right.
$$

To implement the vanishing viscosity method, one needs to show that, as $\mu \downarrow$ 0 , solutions $\left(v_{\mu}, u_{\mu}, s_{\mu}\right)$ of $(1.5)$ converge to functions $(v, u, s)$ which in turn are solutions of (1.1) satisfying the Clausius inequality (1.2). A priori bounds on solutions of (1.5), uniform in $\mu$, are difficult to get so it becomes necessary to carry out this program when we only know that $\left(v_{\mu}, u_{\mu}, s_{\mu}\right)$ converges weakly. The theory of compensated compactness, which may provide the proper vehicle for such a task, has been employed with spectacular success (cf. [Di, C1]) for the system of isentropic flow: $s=\bar{s}=$ constant ,

$$
\left\{\begin{array}{l}
\partial_{t} v-\partial_{x} u=0 \\
\partial_{t} u+\partial_{x} \hat{p}(v, \bar{s})=0 .
\end{array}\right.
$$

It is not clear, however, how to extend this approach to the nonisentropic case, because, in contrast to (1.6), the system (1.1) is not endowed with a rich family of entropy-entropy flux pairs.

The purpose of this paper is to exhibit a class of constitutive relations for which the method of compensated compactness may be applied to (1.1). We introduce a new state vector $(w, u, s)$ and write constitutive equations in the form

$$
v=w+\alpha s, \quad p=h(w), \quad e=H(w)+\beta s, \quad \theta=\alpha h(w)+\beta,
$$


where $h$ is a function with $h^{\prime}<0, \alpha$ is a positive constant, $\beta$ is a constant, and

$$
H(w):=-\int^{w} h(\omega) d \omega .
$$

Of course, (1.7) can be written in the form (1.3) as

$$
e=H(v-\alpha s)+\beta s, \quad p=h(v-\alpha s), \quad \theta=\alpha h(v-\alpha s)+\beta
$$

and (1.4) hold by virtue of (1.8).

The model (1.7) is quite special. Even so, when we are dealing with solutions in which $(v, s)$ do not deviate far from some constant values $(\bar{v}, \bar{s})$ we may obtain a reasonable approximation of general constitutive equations (1.3) by equations of the form (1.9) upon choosing

$$
\begin{gathered}
\alpha=-\hat{p}_{s}(\bar{v}, \bar{s}) / \hat{p}_{v}(\bar{v}, \bar{s}), \quad \beta=\hat{\theta}(\bar{v}, \bar{s})-\alpha \hat{p}(\bar{v}, \bar{s}), \\
h(w)=\hat{p}(w+\alpha \bar{s}, \bar{s})
\end{gathered}
$$

so that $\hat{e}(v, \bar{s})$ and $\hat{p}(v, \bar{s})$ are matched for all $v$ and the values of $\hat{\theta}, \hat{p}_{s}$, and $\hat{\theta}_{v}$ are matched at $(\bar{v}, \bar{s})$. Thus our model may be regarded as a "firstorder correction" to the isentropic model. When we use this to approximate the equations of state of the polytropic gas in the vicinity of any $(\bar{v}, \bar{s})$, it turns out that $\alpha>0, \beta>0$, and $h>0$; hence, in particular, $\theta>0$.

Under the constitutive relations (1.7), the system (1.5) takes the form

$$
\left\{\begin{array}{l}
\partial_{t} w-\partial_{x} u=-\alpha \partial_{t} s \\
\partial_{t} u+\partial_{x} h(w)=\mu \partial_{x}\left(\kappa \partial_{x} u\right) \\
\partial_{t}\left[H(w)+\frac{1}{2} u^{2}\right]+\partial_{x}[u h(w)]=-\beta \partial_{t} s+\mu \partial_{x}\left(\kappa u \partial_{x} u\right)+\mu \partial_{x}\left(\nu \partial_{x} \theta\right)
\end{array}\right.
$$

The special features that make (1.12) tractable by the method of compensated compactness are that the entropy appears linearly and so there is no difficulty in passing to the limit under weakly convergent $s_{\mu}$ while the remaining two fields $(w, u)$ are only weakly coupled to $s$ and may be viewed as solutions of a system of just two equations which is endowed with a rich family of entropy-entropy flux pairs.

For reasons to be explained shortly, we shall also consider a variant of (1.12) which results by adding to $(1.12)_{1}$ artificial viscosity and by selecting in $(1.12)_{3}$ the special function $\nu=-\kappa h / \alpha h^{\prime}$ for the heat conductivity:

(1.13)

$$
\left\{\begin{array}{l}
\partial_{t} w-\partial_{x} u=-\alpha \partial_{t} s+\mu \partial_{x}\left(\kappa \partial_{x} w\right) \\
\partial_{t} u+\partial_{x} h(w)=\mu \partial_{x}\left(\kappa \partial_{x} u\right) \\
\partial_{t}\left[H(w)+\frac{1}{2} u^{2}\right]+\partial_{x}[u h(w)]=-\beta \partial_{t} s+\mu \partial_{x}\left(\kappa u \partial_{x} u\right)-\mu \partial_{x}\left[\kappa h(w) \partial_{x} w\right]
\end{array}\right.
$$

For both systems, (1.12) and (1.13), we assign initial conditions

$$
\begin{aligned}
& w(x, 0)=\bar{w}_{\mu}(x), \quad u(x, 0)=\bar{u}_{\mu}(x), \quad s(x, 0)=\bar{s}_{\mu}(x), \\
& -\infty<x<\infty \text {, }
\end{aligned}
$$

where $\bar{w}_{\mu}, \bar{u}_{\mu}, \bar{s}_{\mu}$ take values in the physical range and satisfy

$$
\begin{gathered}
\left\|\left(\bar{w}_{\mu}, \bar{u}_{\mu}\right)\right\|_{L^{\infty}} \leq C, \quad\left\|\bar{s}_{\mu}\right\|_{L^{1}} \leq C, \\
\int_{-\infty}^{\infty}\left|\bar{w}_{\mu}(x)-w_{\infty}\right|^{2} d x+\int_{-\infty}^{\infty} \bar{u}_{\mu}^{2}(x) d x \leq C
\end{gathered}
$$


for some constant $w_{\infty}$. Here and throughout the paper, $C$ denotes a positive generic constant, independent of $\mu>0$.

In Section 2 we employ the method of compensated compactness to show that when $h$ has at most one inflection point and the solution $\left(w_{\mu}, u_{\mu}, s_{\mu}\right)$ of either (1.12) or (1.13) with initial data (1.14) satisfies $\left\|\left(w_{\mu}, u_{\mu}\right)\right\|_{L^{\infty}} \leq C$, then we have a sequence $\left\{\mu_{n}\right\}, \mu_{n} \rightarrow 0$ as $n \rightarrow \infty, L^{\infty}$ functions $(w, u)$ and a (signed) Radon measure $s$ such that $\left(w_{\mu_{n}}, u_{\mu_{n}}\right) \rightarrow(w, u)$ a.e. and $s_{\mu_{n}} \rightarrow s$ in the space of distributions. Furthermore, if $v=w+\alpha s$ then $(v, u, s)$ is a weak solution of (1.1). Finally, $\partial_{t} s$ is a nonnegative, bounded Radon measure so in particular the Clausius inequality (1.2) is satisfied.

The fact that $\left(w_{\mu_{n}}, u_{\mu_{n}}\right)$, and thereby also $p_{\mu_{n}}=h\left(w_{\mu_{n}}\right)$, converge strongly, no matter how rapidly may the initial data $\left(\bar{w}_{\mu}, \bar{u}_{\mu}, \bar{s}_{\mu}\right)$ oscillate, indicates that the nondegenerate characteristic fields instantaneously quench initial oscillations. Moreover, no rapid oscillations develop as time evolves. On the other hand, one should not expect, in general, strong convergence for $s_{\mu_{n}}$ because initial oscillations may propagate along the linearly degenerate characteristic field associated with $s$ (cf. [C2]).

The result described above hinges upon the assumption $\left\|\left(w_{\mu}, u_{\mu}\right)\right\|_{L^{\infty}} \leq C$. Such an estimate is not currently known for solutions of the system (1.12), with natural, Newtonian viscosity and heat conductivity. However, in Section 3 we will establish the desired $L^{\infty}$ bound for solutions of the system (1.13), with artificial viscosity, under certain assumptions on the function $h$. That we are able to derive these $L^{\infty}$ estimates is the reason for introducing (1.13).

In conclusion, by combining the results of Sections 2 and 3 we establish, in Section 4 , the existence of weak solutions $(v, u, s)$ to (1.1), (1.2), (1.9) such that $u$ and $v-\alpha s$ are in $L^{\infty}$ and $s$ is a Radon measure.

\section{COMPACTNESS IN THE FRAMEWORK OF THE VANISHING VISCOSITY METHOD}

In this section we investigate the limit behavior, as $\mu \downarrow 0$, of solutions of the systems (1.12) and (1.13), under initial conditions (1.14) satisfying (1.15), (1.16). The function $h$ has at most one inflection point. The initial data take values in the physical range so in particular we require

$$
\theta_{\infty}:=\alpha h\left(w_{\infty}\right)+\beta>0
$$

We begin with the prototypical system

$$
\left\{\begin{array}{l}
\partial_{t} w-\partial_{x} u=0 \\
\partial_{t} u+\partial_{x} h(w)=0
\end{array}\right.
$$

The entropy-entropy flux pairs $(\eta, q)$ for (2.2) (cf. [La]) are governed by

$$
q_{w}=h^{\prime}(w) \eta_{u}, \quad q_{u}=-\eta_{w}
$$

whence

$$
\eta_{w w}+h^{\prime}(w) \eta_{u u}=0 .
$$

Then we have the following result of DiPerna [Di]:

Proposition 2.1. Let $\left(w_{\mu}, u_{\mu}\right), \mu>0$, be a family of measurable functions satisfying

$$
\left\|\left(w_{\mu}, u_{\mu}\right)\right\|_{L^{\infty}} \leq C .
$$


Assume further that for any entropy-entropy flux pair $(\eta, q)$ of $(2.2)$

$$
\partial_{t} \eta\left(w_{\mu}, u_{\mu}\right)+\partial_{x} q\left(w_{\mu}, u_{\mu}\right) \subset \text { compact subset of } H^{-1} .
$$

Then there is a sequence $\left\{\mu_{n}\right\}, \mu_{n} \rightarrow 0$ as $n \rightarrow \infty$, and $L^{\infty}$ functions $(w, u)$ such that

$$
\left(w_{\mu_{n}}, u_{\mu_{n}}\right) \rightarrow(w, u), \quad n \rightarrow \infty \text {, a.e. }
$$

With the help of the above proposition we will establish compactness in the framework of our two vanishing viscosity schemes.

2.1. Vanishing Newtonian viscosity. Here our governing system will be (1.5) in its form (1.12). The viscosity $\kappa=\hat{\kappa}(v, s)$ and heat conductivity $\nu=\hat{\nu}(v, s)$ will be fixed bounded, continuous, nonnegative functions that are comparable on the range of solutions:

$$
m \hat{\kappa}(v, s) \leq \hat{\nu}(v, s) \leq M \hat{\kappa}(v, s)
$$

for positive constants $m$ and $M$.

Combining the equations in (1.5) yields the identity

$$
\partial_{t} s=\frac{\mu \kappa}{\theta}\left(\partial_{x} u\right)^{2}+\frac{\mu \nu}{\theta^{2}}\left(\partial_{x} \theta\right)^{2}+\mu \partial_{x}\left(\frac{\nu}{\theta} \partial_{x} \theta\right)
$$

which is of course compatible with the Clausius-Duhem inequality. Similarly, by virtue of (1.12), (1.8), and (2.1),

(2.10)

$$
\begin{aligned}
& \partial_{t}\left[H(w)-H\left(w_{\infty}\right)-H^{\prime}\left(w_{\infty}\right)\left(w-w_{\infty}\right)+\frac{1}{2} u^{2}\right]+\partial_{x}\left[u\left(h(w)-h\left(w_{\infty}\right)\right)\right] \\
& \quad=-\theta_{\infty} \partial_{t} s+\mu \partial_{x}\left[\kappa u \partial_{x} u+\nu \partial_{x} \theta\right] .
\end{aligned}
$$

Theorem 2.1. Let $\left(w_{\mu}, u_{\mu}, s_{\mu}\right), \mu>0$, be solutions of $(1.12)$ on $(-\infty, \infty) \times$ $[0, T)$ with initial data (1.14) satisfying (1.15), (1.16), and (2.1). Assume

$$
\begin{gathered}
\left\|\left(w_{\mu}, u_{\mu}\right)\right\|_{L^{\infty}} \leq C, \\
\theta_{\mu}=\alpha h\left(w_{\mu}\right)+\beta>0 .
\end{gathered}
$$

Then we have a sequence $\left\{\mu_{n}\right\}, \mu_{n} \rightarrow 0$ as $n \rightarrow \infty, L^{\infty}$ functions $(w, u)$, and $a$ (signed) Radon measure $s$ such that

$$
\begin{gathered}
\left(w_{\mu_{n}}, u_{\mu_{n}}\right) \rightarrow(w, u), \quad n \rightarrow \infty, \text { a.e. } \\
s_{\mu_{n}} \rightarrow s, \quad n \rightarrow \infty, \quad \text { in } \mathscr{D}^{\prime} .
\end{gathered}
$$

Furthermore, if $v=w+\alpha s,(v, u, s)$ is a weak solution of (1.1), (1.9). Finally, $\partial_{t} s$ is a nonnegative, bounded Radon measure and thus the Clausius inequality (1.2) is satisfied.

Proof. From (2.10) and (2.9) it follows

$$
\begin{aligned}
\int_{-\infty}^{\infty}[ & \left.H\left(w_{\mu}(x, t)\right)-H\left(w_{\infty}\right)-H^{\prime}\left(w_{\infty}\right)\left(w_{\mu}(x, t)-w_{\infty}\right)+\frac{1}{2} u_{\mu}^{2}(x, t)\right] d x \\
& +\theta_{\infty} \int_{0}^{t} \int_{-\infty}^{\infty}\left[\frac{\mu \kappa}{\theta_{\mu}}\left(\partial_{x} u_{\mu}\right)^{2}+\frac{\mu \nu}{\theta_{\mu}^{2}}\left(\partial_{x} \theta_{\mu}\right)^{2}\right] d x d \tau \\
= & \int_{-\infty}^{\infty}\left[H\left(\bar{w}_{\mu}(x)\right)-H\left(w_{\infty}\right)-H^{\prime}\left(w_{\infty}\right)\left(\bar{w}_{\mu}(x)-w_{\infty}\right)+\frac{1}{2} \bar{u}_{\mu}^{2}(x)\right] d x
\end{aligned}
$$


whence, upon using (1.16) and (2.1),

$$
\left\|\left(\frac{\mu \kappa}{\theta_{\mu}}\right)^{1 / 2} \partial_{x} u_{\mu}\right\|_{L^{2}} \leq C, \quad\left\|\left(\frac{\mu \nu}{\theta_{\mu}^{2}}\right)^{1 / 2} \partial_{x} \theta_{\mu}\right\|_{L^{2}} \leq C .
$$

By virtue of (1.12) and (2.9), $\left(w_{\mu}, u_{\mu}\right)$ satisfy the system of two equations

$$
\left\{\begin{array}{l}
\partial_{t} w_{\mu}-\partial_{x} u_{\mu}=-\frac{\alpha \mu \kappa}{\theta_{\mu}}\left(\partial_{x} u_{\mu}\right)^{2}-\frac{\alpha \mu \nu}{\theta_{\mu}^{2}}\left(\partial_{x} \theta_{\mu}\right)^{2}-\mu \partial_{x}\left(\frac{\alpha \nu}{\theta_{\mu}} \partial_{x} \theta_{\mu}\right), \\
\partial_{t} u_{\mu}+\partial_{x} h\left(w_{\mu}\right)=\mu \partial_{x}\left(\kappa \partial_{x} u_{\mu}\right) .
\end{array}\right.
$$

Let $(\eta, q)$ be any entropy-entropy flux pair for (2.2). We multiply $(2.17)_{1}$ by $\eta_{w},(2.17)_{2}$ by $\eta_{u}$; we add the resulting equations and use (2.3) to get

$$
\partial_{t} \eta\left(w_{\mu}, u_{\mu}\right)+\partial_{x} q\left(w_{\mu}, u_{\mu}\right)=I_{\mu}+J_{\mu},
$$

where

$$
\begin{aligned}
I_{\mu}= & -\left[\theta_{\mu} \eta_{u u}+\alpha \eta_{w}\right] \frac{\mu \kappa}{\theta}\left(\partial_{x} u_{\mu}\right)^{2}+\left[\frac{\theta_{\mu}}{h^{\prime}} \eta_{w w}-\alpha \eta_{w}\right] \frac{\mu \nu}{\theta_{\mu}^{2}}\left(\partial_{x} \theta_{\mu}\right)^{2} \\
& +\eta_{w u}\left[\alpha \nu-\frac{\theta_{\mu}}{\alpha h^{\prime}} \kappa\right] \frac{\mu}{\theta_{\mu}}\left(\partial_{x} u_{\mu}\right)\left(\partial_{x} \theta_{\mu}\right),
\end{aligned}
$$

$$
J_{\mu}=\partial_{x}\left[\eta_{u} \mu \kappa \partial_{x} u_{\mu}-\alpha \eta_{w} \frac{\mu \nu}{\theta_{\mu}} \partial_{x} \theta_{\mu}\right] .
$$

By virtue of (2.11), (2.12), (2.16), and (2.8),

$$
\begin{aligned}
\left\|I_{\mu}\right\|_{L^{1}} & \leq C, \\
J_{\mu} \rightarrow 0, \quad \mu & \rightarrow 0, \text { in } H^{-1} .
\end{aligned}
$$

It follows that the family $I_{\mu}+J_{\mu}$, and thereby also the left-hand side of (2.18), for any $\mu>0$, are contained in a compact subset of $W^{-1, p}, 1 \leq p<2$. On the other hand, by (2.11), the left-hand side of (2.18) is bounded in $W^{-1, q}, 2<$ $q \leq \infty$, uniformly in $\mu$. The generalized version (cf. [C1]) of Murat's lemma (cf. [Ta]) asserts that compactness in $W^{-1, p}, 1 \leq p<2$, together with boundedness in $W^{-1, q}, 2<q \leq \infty$, implies compactness in $H^{-1}$. Thus the hypothesis (2.6) of Proposition 2.1 is satisfied. Consequently, there is a sequence $\left\{\mu_{n}\right\}, \mu_{n} \rightarrow 0$ as $n \rightarrow \infty$, and $L^{\infty}$ functions $(w, u)$ such that (2.13) holds.

Similarly, by virtue of (2.16), the first two terms on the right-hand side of (2.9) are uniformly bounded in $L^{1}$ while the third term tends to zero in $H^{-1}$, as $\mu \rightarrow 0$. It follows that the family $\partial_{t} s_{\mu}$ is contained in a compact subset of $W^{-1, p}, 1 \leq p<2$. The same is true for the family $s_{\mu}$. This yields (2.14). From (2.9) it follows that $\partial_{t} s$ is a nonnegative distribution and thereby a nonnegative Radon measure, which is bounded by account of (2.15). Recalling (1.15) we conclude that $s$ is a (signed) Radon measure.

Let us set $v=w+\alpha s$. Combining (2.13), (2.14), and (2.16), we verify that $(v, u, s)$ is a distributional solution of (1.1). The proof is complete.

2.2. Vanishing artificial viscosity. We now turn to system (1.13). We easily verify the identity

$$
\partial_{t} s=\frac{\mu \kappa}{\theta}\left(\partial_{x} u\right)^{2}-\frac{\mu \kappa h^{\prime}}{\theta}\left(\partial_{x} w\right)^{2},
$$


which is the analog of (2.9). Similarly, in the place of (2.10) we now have (2.24)

$$
\begin{gathered}
\partial_{t}\left[H(w)-H\left(w_{\infty}\right)-H^{\prime}\left(w_{\infty}\right)\left(w-w_{\infty}\right)+\frac{1}{2} u^{2}\right]+\partial_{x}\left[u\left(h(w)-h\left(w_{\infty}\right)\right)\right] \\
\quad=-\theta_{\infty} \partial_{t} s+\mu \partial_{x}\left[\kappa u \partial_{x} u-\frac{\kappa}{\alpha}\left(\theta-\theta_{\infty}\right) \partial_{x} w\right] .
\end{gathered}
$$

Theorem 2.2. Let $\left(w_{\mu}, u_{\mu}, s_{\mu}\right), \mu>0$, be solutions of $(1.13)$ on $(-\infty, \infty) \times$ $[0, T)$ with initial data (1.14) satisfying (1.15), (1.16), and (2.1). Assume

$$
\begin{gathered}
\left\|\left(w_{\mu}, u_{\mu}\right)\right\|_{L^{\infty}} \leq C, \\
\theta_{\mu}=\alpha h\left(w_{\mu}\right)+\beta>0 .
\end{gathered}
$$

Then we have a sequence $\left\{\mu_{n}\right\}, \mu_{n} \rightarrow 0$ as $n \rightarrow \infty, L^{\infty}$ functions $(w, u)$, and a (signed) Radon measure $s$ such that

$$
\begin{gathered}
\left(w_{\mu_{n}}, u_{\mu_{n}}\right) \rightarrow(w, u), \quad n \rightarrow \infty, \text { a.e. } \\
s_{\mu_{n}} \rightarrow s, \quad n \rightarrow \infty, \text { in } \mathscr{D}^{\prime} .
\end{gathered}
$$

Furthermore, if $v=w+\alpha s,(v, u, s)$ is a weak solution of (1.1), (1.9). Finally, $\partial_{t} s$ is a nonnegative, bounded Radon measure and thus the Clausius inequality (1.2) is satisfied.

Proof. From (2.24) and (2.23) we obtain

$$
\begin{aligned}
& \int_{-\infty}^{\infty} {\left[H\left(w_{\mu}(x, t)\right)-H\left(w_{\infty}\right)-H^{\prime}\left(w_{\infty}\right)\left(w_{\mu}(x, t)-w_{\infty}\right)+\frac{1}{2} u_{\mu}^{2}(x, t)\right] d x } \\
& \quad+\theta_{\infty} \int_{0}^{t} \int_{-\infty}^{\infty}\left[\frac{\mu \kappa}{\theta_{\mu}}\left(\partial_{x} u_{\mu}\right)^{2}-\frac{\mu \kappa h^{\prime}}{\theta_{\mu}}\left(\partial_{x} w_{\mu}\right)^{2}\right] d x d \tau \\
& \quad=\int_{-\infty}^{\infty}\left[H\left(\bar{w}_{\mu}(x)\right)-H\left(w_{\infty}\right)-H^{\prime}\left(w_{\infty}\right)\left(\bar{w}_{\mu}(x)-w_{\infty}\right)+\frac{1}{2} \bar{u}_{\mu}^{2}(x)\right] d x,
\end{aligned}
$$

whence, upon using (1.16), (2.1), (2.23), and $h^{\prime}<0$,

$$
\left\|\left(\frac{\mu \kappa}{\theta_{\mu}}\right)^{1 / 2} \partial_{x} w_{\mu}\right\|_{L^{2}} \leq C, \quad\left\|\left(\frac{\mu \kappa}{\theta_{\mu}}\right)^{1 / 2} \partial_{x} u_{\mu}\right\|_{L^{2}} \leq C,
$$

By account of (1.13) and (2.23), $\left(w_{\mu}, u_{\mu}\right)$ satisfy the system of two equations

$$
\left\{\begin{array}{l}
\partial_{t} w_{\mu}-\partial_{x} u_{\mu}=-\frac{\alpha \mu \kappa}{\theta_{\mu}}\left(\partial_{x} u_{\mu}\right)^{2}+\frac{\alpha \mu \kappa h^{\prime}}{\theta_{\mu}}\left(\partial_{x} w_{\mu}\right)^{2}+\mu \partial_{x}\left(\kappa \partial_{x} w_{\mu}\right), \\
\partial_{t} u_{\mu}+\partial_{x} h\left(w_{\mu}\right)=\mu \partial_{x}\left(\kappa \partial_{x} u_{\mu}\right) .
\end{array}\right.
$$

Let $(\eta, q)$ be any entropy-entropy flux pair for (2.2). We multiply $(2.32)_{1}$ by $\eta_{w},(2.32)_{2}$ by $\eta_{u}$; we add the resulting equations and use (2.3) to get

$$
\begin{aligned}
\partial_{t} \eta\left(w_{\mu}, u_{\mu}\right)+\partial_{x} q\left(w_{\mu}, u_{\mu}\right) \\
=\partial_{x}\left[\eta_{w} \mu \kappa \partial_{x} w_{\mu}+\eta_{u} \mu \kappa \partial_{x} u_{\mu}\right]-\left[\theta_{\mu} \eta_{w w}-\alpha h^{\prime} \eta_{w}\right] \frac{\mu \kappa}{\theta_{\mu}}\left(\partial_{x} w_{\mu}\right)^{2} \\
-\left[\theta_{\mu} \eta_{u u}+\alpha \eta_{w}\right] \frac{\mu \kappa}{\theta_{\mu}}\left(\partial_{x} u_{\mu}\right)^{2}-2 \eta_{w u} \mu \kappa\left(\partial_{x} w_{\mu}\right)\left(\partial_{x} u_{\mu}\right) .
\end{aligned}
$$


Retracing now the steps in the proof of Theorem 2.1, we show, with the help of (2.33) and (2.30), that the hypothesis (2.6) of Proposition 2.1 is satisfied and thus we do have a sequence $\left\{\mu_{n}\right\}, \mu_{n} \rightarrow 0$ as $n \rightarrow \infty$, and $L^{\infty}$ functions $(w, u)$ so that $(2.27)$ holds.

To get (2.28), we appeal to weak compactness in the space of Radon measures (cf. [E]), noting that by (2.31) and (1.15) $\left\|s_{\mu}\right\|_{L^{1}} \leq C(1+T)$. On account of (2.31), $\partial_{t} s$ is a nonnegative, bounded Radon measure.

Finally, we set $v=w+\alpha s$ and use (2.27), (2.28), (2.30), and (2.31) to show that $(v, u, s)$ is a weak solution of (1.1), satisfying the Clausius inequality (1.2). The proof is complete.

\section{3. $L^{\infty}$ ESTIMATES}

We consider solutions $\left(w_{\mu}, u_{\mu}, s_{\mu}\right)$ of $(1.13)$ on $(-\infty, \infty) \times[0, T)$ with initial data (1.14) satisfying (1.15), (1.16), and (2.1) and derive $L^{\infty}$ bounds on $\left(w_{\mu}, u_{\mu}\right)$, independent of $\mu>0$. We assume a priori that our solution satisfies $\theta_{\mu}>0$; our estimates will justify this, a posteriori.

As noted before, $\left(w_{\mu}, u_{\mu}\right)$ is a solution of the system (2.32) in which $s$ enters only through $\kappa$. In the isentropic case, $\alpha=0$, it is known (cf. [CCS]) that solutions of (2.32) have invariant regions, provided that $h$ has a unique inflection point $\hat{w}: h^{\prime \prime}(w) \geq 0$ for $w<\hat{w}, h^{\prime \prime}(w) \leq 0$ for $w>\hat{w}$. In the present case, $\alpha>0$, the appropriate assumption on $h$ is that, for some $\hat{w}$,

$$
h^{\prime \prime}(w)-4 \frac{\alpha h^{\prime}(w)^{2}}{\alpha h(w)+\beta} \begin{cases}\geq 0, & \text { if } w<\hat{w}, \\ \leq 0, & \text { if } w>\hat{w} .\end{cases}
$$

We introduce the notation

$$
g(w):=\sqrt{-h^{\prime}(w)}
$$

and define the classical Riemann invariants

$$
r_{ \pm}(w, u)=u \pm \int_{\hat{w}}^{w} g(\omega) d \omega .
$$

Theorem 3.1. Under the assumption (3.1) and for any $\rho>0$, the domain $\mathscr{R}=$ $\left\{(w, u):-\rho \leq r_{-} \leq \rho,-\rho \leq r_{+} \leq \rho\right\}$ is a positively invariant region for solutions $\left(w_{\mu}, u_{\mu}\right)$ of $(2.32)$.

Proof. If $\left(w_{\mu}, u_{\mu}, s_{\mu}\right)$ is a classical solution of (1.13), the invariance of $\mathscr{R}$ is a consequence of the maximum principle (cf. [CCS]) upon observing that (3.3), (3.2), and (2.32) imply the identities

$$
\begin{aligned}
\partial_{t} r_{ \pm} \mp g \partial_{x} r_{ \pm} & =\mu \partial_{x}\left(\kappa \partial_{x} r_{ \pm}\right) \mp g \frac{\alpha \kappa}{\theta}\left(\partial_{x} r_{ \pm}\right)^{2} \\
& \mp \mu h^{\prime} \frac{2 \alpha \kappa}{\theta_{\mu}}\left(\partial_{x} w_{\mu}\right)\left(\partial_{x} r_{ \pm}\right) \pm \mu \frac{\kappa}{2 g}\left[h^{\prime \prime}-4 \frac{\alpha h^{\prime 2}}{\alpha h+\beta}\right]\left(\partial_{x} w_{\mu}\right)^{2}
\end{aligned}
$$

We now sketch an alternative proof, following [Da], which is lengthier but applies even when $\left(w_{\mu}, u_{\mu}, s_{\mu}\right)$ is a weak solution of (1.13), with $w_{\mu}$ and $u_{\mu}$ merely Lipschitz continuous. We recall that if $(\eta, q)$ is any entropy-entropy flux pair for (2.2), then $\left(w_{\mu}, u_{\mu}\right)$ satisfy the identity (2.33). We seek $\eta$ for which the quadratic form in $\left(\partial_{x} w_{\mu}, \partial_{x} u_{\mu}\right)$ on the right-hand side of $(2.33)$ is 
nonpositive. By account of (2.4) and (3.2), the condition on the discriminant of the quadratic form becomes

$$
g^{2}\left[\eta_{u u}+\frac{\alpha}{\theta} \eta_{w}\right]^{2}-\eta_{w u}^{2} \geq 0
$$

We construct solutions of $(2.4)$ :

$$
\eta=Y(w) \cosh (k u),
$$

where $Y$ is the solution of the initial-value problem

$$
\begin{gathered}
Y^{\prime \prime}-k^{2} g^{2} Y=0, \\
Y(\hat{w})=1, \quad Y^{\prime}(\hat{w})=0 .
\end{gathered}
$$

For $\eta$ of the form (3.6) and $k \gg 0,(3.5)$ will hold provided

$$
\chi_{+}(w) \geq 0, \quad \chi_{-}(w) \geq 0, \quad \text { for all } w,
$$

where

$$
\chi_{ \pm}:=k g Y+\frac{1}{k} g \frac{\alpha}{\theta} Y^{\prime} \pm Y^{\prime} .
$$

Using (3.10) and (3.7) and after a lengthy but straightforward calculation:

$$
\chi_{ \pm}^{\prime} \pm \frac{k h^{\prime}+\frac{1}{2 k} \frac{\alpha}{\theta}\left[h^{\prime \prime}-\frac{2 \alpha}{\theta} h^{\prime 2}\right]}{g \mp \frac{1}{k} \frac{\alpha}{\theta} h^{\prime}} \chi_{ \pm}=-\frac{k}{2} \frac{h^{\prime \prime}-4 \frac{\alpha}{\theta} h^{\prime 2}}{g \mp \frac{1}{k} \frac{\alpha}{\theta} h^{\prime}} Y .
$$

Assume $k \gg 0$. By virtue of $(3.1),(1.7)$ and since $\chi_{ \pm}(\hat{w})=k g(\hat{w})>0,(3.11)$ implies (3.9).

Once we have secured that the quadratic form on the right-hand side of (2.33) is nonpositive, we show that

$$
\begin{aligned}
\int_{-\infty}^{\infty}\left[Y\left(w_{\mu}(x, t)\right) \cosh \left(k u_{\mu}(x, t)\right)-Y\left(w_{\infty}\right)-Y^{\prime}\left(w_{\infty}\right)\left(w_{\mu}(x, t)-w_{\infty}\right)\right] d x \\
\leq \int_{-\infty}^{\infty}\left[Y\left(\bar{w}_{\mu}(x)\right) \cosh \left(k \bar{u}_{\mu}(x)\right)-Y\left(w_{\infty}\right)-Y^{\prime}\left(w_{\infty}\right)\left(\bar{w}_{\mu}(x)-w_{\infty}\right)\right] d x \\
+\frac{\alpha}{\theta_{\infty}}\left|Y^{\prime}\left(w_{\infty}\right)\right| \int_{-\infty}^{\infty}\left[H\left(\bar{w}_{\mu}(x)\right)-H\left(w_{\infty}\right)\right. \\
\left.-H^{\prime}\left(w_{\infty}\right)\left(\bar{w}_{\mu}(x)-w_{\infty}\right)+\frac{1}{2} \bar{u}_{\mu}^{2}(x)\right] d x .
\end{aligned}
$$

To see this, write (2.33) with $\eta=Y(w) \cosh (k u)-Y\left(w_{\infty}\right)+Y^{\prime}\left(w_{\infty}\right) w_{\infty}$, multiply $(2.32)_{1}$ by $-Y^{\prime}\left(w_{\infty}\right)$, add the two equations, integrate the resulting equation over $(-\infty, \infty) \times[0, t]$, and note that the integral of the right-hand side of (2.33) will be nonpositive while the integral of the right-hand side of $(2.32)_{1}$ may be bounded with the help of (2.29).

The invariant regions are now established by raising (3.12) to the power $1 / k$ and letting $k \rightarrow \infty$, because an elementary analysis of the asymptotic form of the solutions of (3.7), (3.8) for large $k$ (cf. [Da]) shows

$$
\lim _{k \rightarrow \infty} \eta(w, u)^{1 / k}= \begin{cases}\exp \left[r_{+}(w, u)\right] & \text { if } w>\hat{w}, u>0 \\ \exp \left[r_{-}(w, u)\right] & \text { if } w<\hat{w}, u>0 \\ \exp \left[-r_{+}(w, u)\right] & \text { if } w<\hat{w}, u<0 \\ \exp \left[-r_{-}(w, u)\right] & \text { if } w>\hat{w}, u<0\end{cases}
$$

This completes the proof of the theorem. 


\section{EXISTENCE OF SOLUTIONS TO THE HYPERBOLIC SYSTEM}

Here we combine the results of the previous sections to establish the existence of solutions to the initial-value problem for the system of conservation laws of mass, momentum, and energy in the special class of one-dimensional thermoelastic media under consideration.

We consider the system (1.1) with constitutive equations (1.9), and (1.8), where $h$ is a smooth, strictly decreasing function, $h^{\prime}(w)<0$, with at most one inflection point and satisfying (3.1) for some $\hat{w}$.

We prescribe initial conditions

$$
(v(\cdot, 0), u(\cdot, 0), s(\cdot, 0))=\left(v_{0}, u_{0}, s_{0}\right)
$$

on $(-\infty, \infty)$, with the following properties:

(i) $u_{0}$ is in $L^{\infty}(\mathbb{R}) ; v_{0}$ and $s_{0}$ are Radon measures on $\mathbb{R}$; however, $w_{0}:=$ $v_{0}-\alpha s_{0}$ is in $L^{\infty}(\mathbb{R})$.

(ii) $u_{0}$ is in $L^{2}(\mathbb{R}) ; w_{0}-w_{\infty}$ is in $L^{2}(\mathbb{R})$, for some constant $w_{\infty} ; s_{0}$ is a bounded Radon measure on $\mathbb{R}$.

(iii) The closed convex hull of the range of $\left(w_{0}, u_{0}\right)$ is contained in a region $\mathscr{R}=\left\{(w, u):-\rho \leq r_{-} \leq \rho,-\rho \leq r_{+} \leq \rho\right\}$ (see (3.3)) which contains only physically admissible states. In particular, if $(w, u) \in \mathscr{R}$ then $\theta=\alpha h(w)+\beta>0$.

Theorem 4.1. Under the above assumptions on $h$ and the initial data, there exists a distributional solution $(v, u, s)$ of $(1.1),(4.1)$ on $(-\infty, \infty) \times[0, \infty)$ such that $u$ is in $L^{\infty}, v$ and $s$ are Radon measures and $w:=v-\alpha s$ is in $L^{\infty}$. The range of $(w, u)$ is contained in the region $\mathscr{R}$. For each fixed $t \in[0, \infty), u(\cdot, t)$ and $w(\cdot, t)-w_{\infty}$ are bounded in $L^{2}(\mathbb{R})$ and $s(\cdot, t)$ is a bounded Radon measure on $\mathbb{R}$, uniformly in $t$. Furthermore, $\partial_{t} s$ is a bounded nonnegative Radon measure and thus the Clausius inequality (1.2) is satisfied.

Proof. We take any standard, $C^{\infty}$, nonnegative mollifier $\psi$ on $\mathbb{R}$ and for $\mu>0$ we form the convolution of $\left(w_{0}, u_{0}, s_{0}\right)$ with $\psi_{\mu}(x):=\mu^{-1} \psi(x / \mu)$ thus obtaining a family $\left(\bar{w}_{\mu}, \bar{u}_{\mu}, \bar{s}_{\mu}\right)$ of $C^{\infty}$ functions on $\mathbb{R}$ with the following properties: (i) as $\mu \downarrow 0,\left(\bar{w}_{\mu}, \bar{u}_{\mu}\right) \rightarrow\left(w_{0}, u_{0}\right)$ in $L^{\infty}$ weak $^{*}, \bar{s}_{\mu} \rightarrow s_{0}$ in $\mathscr{M},\left(\bar{w}_{\mu}-w_{\infty}, \bar{u}_{\mu}\right) \rightarrow\left(w_{0}-w_{\infty}, u_{0}\right)$ in $L^{2}$; (ii) (1.15) and (1.16) hold; (iii) the range of $\left(\bar{w}_{\mu}, \bar{u}_{\mu}\right)$ is contained in the region $\mathscr{R}$.

For any $\mu>0$, we can solve, by the energy method, the initial-value problem (1.13), (1.14), for instance with $\kappa \equiv 1$, and construct the family $\left(w_{\mu}, u_{\mu}, s_{\mu}\right)$ of solutions on $(-\infty, \infty) \times[0, \infty)$. On account of Theorem 3.1, the range of $\left(w_{\mu}, u_{\mu}\right)$ is contained in the region $\mathscr{R}$. Thus, the hypotheses (2.25) and (2.26) are satisfied in which case Theorem 2.2 yields the solution $(v, u, s)$ of $(1.1)$. It is easy to verify that $(v, u, s)$ satisfies the initial conditions (4.1) (in the weak sense). Clearly, $(w, u)$ takes values in $\mathscr{R}$. By virtue of (2.29), (1.15), and $(2.31)$, for any $t \in[0, \infty)$,

$$
\begin{gathered}
\int_{-\infty}^{\infty}\left|w_{\mu}(x, t)-w_{\infty}\right|^{2} d x+\int_{-\infty}^{\infty} u_{\mu}^{2}(x, t) d x \leq C, \\
\int_{-\infty}^{\infty}\left|s_{\mu}(x, t)\right| d x \leq C
\end{gathered}
$$


whence we deduce that $u(\cdot, t)$ and $w(\cdot, t)-w_{\infty}$ are bounded in $L^{2}(\mathbb{R})$ and $s(\cdot, t)$ is a bounded Radon measure on $\mathbb{R}$. The proof is complete.

Remark 4.1. Additional restrictions on solutions of (1.1) dictated by physics may be handled by suitable hypotheses. For example, if $v$ stands for specific volume we need $v>0$. This may be attained by requiring $\alpha s_{0}>-w_{\min }$ where $w_{\text {min }}=\min \{w:(w, u) \in \mathscr{R}\}$.

Remark 4.2. Because $\partial_{t} s$ is always a positive Radon measure, if $s_{0} \geq 0$ then the entropy $s$ will also be a positive Radon measure.

\section{REFERENCES}

[C1] G.-Q. Chen, The compensated compactness method and the system of isentropic gas dynamics, MSRI Preprint 00527-91 (1990).

[C2] - The method of quasidecoupling for discontinuous solutions to conservation laws, Arch. Rational Mech. Anal. 121 (1992), 131-185.

[CCS] K. N. Chueh, C. C. Conley, and J. A. Smoller, Positively invariant regions for systems of nonlinear diffusion equations, Indiana Univ. Math. J. 26 (1977), 372-411.

[Da] C. M. Dafermos, Estimates for conservation laws with little viscosity, SIAM J. Math. Anal. 18 (1987), 409-421.

[Di] R. J. DiPerna, Convergence of approximate solutions to conservation laws, Arch. Rational Mech. Anal. 82 (1983), 27-70.

[E] L. C. Evans and R. Gariepy, Measure theory and fine properties of functions, CRC Press, Ann Arbor, MI, 1992.

[Gl] J. Glimm, Solutions in the large for nonlinear hyperbolic systems of equations, Comm. Pure Appl. Math. 18 (1965), 697-715.

[La] P. D. Lax, Shock waves and entropy, Contributions to Functional Analysis (E. A. Zarantonello, ed.), Academic Press, New York, 1971, pp. 603-634.

[Li] T.-P. Liu, Solutions in the large for the equations of nonisentropic gas dynamics, Indiana Univ. Math. J. 26 (1977), 147-177.

[M] F. Murat, L'injection du cone positif de $H^{-1}$ dans $W^{-1, q}$ est compacte pur tout $q<2$, J. Math. Pures Appl. 60 (1981), 309-322.

[Ta] L. Tartar, Compensated compactness and applications to partial differential equations, Pitman Res. Notes Math., vol. 39, Nonlinear Analysis and Mechanics (R. J. Knops, ed.), Pitman Press, New York, 1979.

Department of Mathematics, The University of Chicago, Chicago, Illinois 60637

E-mail address: cheng@zaphod.uchicago.edu

Division of Applied Mathematics, Brown University, Providence, Rhode Island 02912 